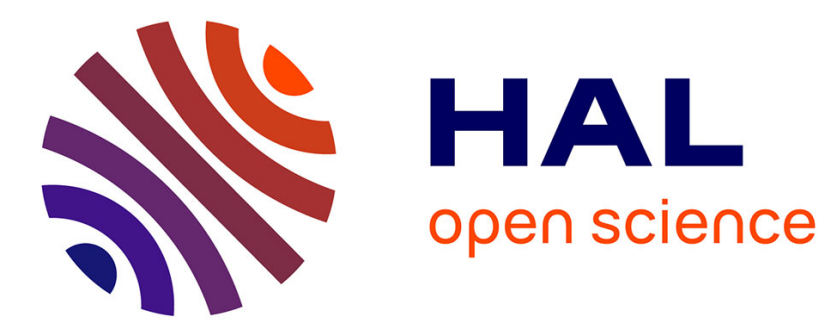

\title{
Applicability of RAD-tag genotyping for inter-familial comparisons: empirical data from two cetaceans
}

\author{
Amélia Viricel, Eric Pante, Willy Dabin, Benoit Simon-Bouhet
}

\section{To cite this version:}

Amélia Viricel, Eric Pante, Willy Dabin, Benoit Simon-Bouhet. Applicability of RAD-tag genotyping for inter-familial comparisons: empirical data from two cetaceans. Molecular Ecology Resources, 2014, 14 (3), pp.597-605. 10.1111/1755-0998.12206 . hal-00908459

\section{HAL Id: hal-00908459 \\ https://hal.science/hal-00908459}

Submitted on 23 Nov 2013

HAL is a multi-disciplinary open access archive for the deposit and dissemination of scientific research documents, whether they are published or not. The documents may come from teaching and research institutions in France or abroad, or from public or private research centers.
L'archive ouverte pluridisciplinaire HAL, est destinée au dépôt et à la diffusion de documents scientifiques de niveau recherche, publiés ou non, émanant des établissements d'enseignement et de recherche français ou étrangers, des laboratoires publics ou privés. 


\section{Applicability of RAD-tag genotyping for inter-familial comparisons: empirical data from two cetaceans}

Amélia Viricel ${ }^{1}$, Eric Pante ${ }^{1}$, Willy Dabin ${ }^{2}$, and Benoit Simon-Bouhet ${ }^{1}$.

1 Littoral, Environnement et Sociétés (LIENSs) UMR 7266 CNRS, Université de La

$5 \quad$ Rochelle, 2 rue Olympe de Gouges, 17000 La Rochelle, France

62 Observatoire PELAGIS, UMS 3462 CNRS, Université de La Rochelle, Pôle analytique,

75 allées de l'océan, 17000 La Rochelle, France

$8 \quad *$ E-mail: amelia.viricel@gmail.com ; Phone: +33.05.46.50.76.42 ; Fax: +33.05.46.50.76.63.

$9 \quad$ Running Title: RAD-tag genotyping across cetacean families

10 Keywords: RAD sequencing ; phylogenetics ; genomics ; inter-familial divergence ; Delphinidae ;

11 Phocoenidae 
12

\section{Abstract}

Restriction site-Associated DNA tag (RAD-tag) sequencing has become a popular approach to generate thousands of SNPs used to address diverse questions in population genomics. Comparatively, the suitability of RAD-tag genotyping to address evolutionary questions across divergent species has been the subject of only a few recent studies. Here, we evaluate the applicability of this approach to conduct genome-wide scans for polymorphisms across two cetacean species belonging to distinct families: the short-beaked common dolphin (Delphinus delphis; $\mathrm{n}=5$ individuals) and the harbor porpoise (Phocoena phocoena $; \mathrm{n}=1$ individual). Additionally, we explore the effects of varying two parameters in the Stacks analysis pipeline on the number of loci and level of divergence obtained. We observed a $34 \%$ drop in the total number of loci that were present in all individuals when analyzing individuals from the distinct families compared to analyses restricted to intra-specific comparisons (i.e., within D. delphis). Despite relatively stringent quality filters, 3,595 polymorphic loci were retrieved from our inter-familial comparison. Cetaceans have undergone rapid diversification and the estimated divergence time between the two families is relatively recent (14 to $19 \mathrm{My}$ ). Thus, our results showed that, for this level of divergence, a large number of orthologous loci can still be genotyped using this approach, which is on par with two recent in silico studies. Our findings constitute one of the first empirical investigations using RAD-tag sequencing at this level of divergence and highlights the great potential of this approach in comparative studies and to address evolutionary questions. 


\section{Introduction}

Recent parallel DNA sequencing technologies have enabled population genomics studies in non-model organisms including characterizing patterns of hybridization and introgression (e.g. Hohenlohe et al. 2011), intra-specific phylogeography (e.g. Emerson et al. 2010), QTL mapping (e.g. Gagnaire et al. 2013) and studying the genetic basis of adaptations (Stapley et al., 2010). There is now a growing interest in these methods in the fields of biogeography (Lexer et al., 2013) and phylogenetics (McCormack et al., 2013).

Among recent genotyping methods using next-generation sequencing, Restriction site-Associated DNA tag (RAD-tag) sequencing has become one of the most popular approaches to conduct population genomics studies in non-model organisms. To date, however, few studies have explored the applicability of this approach to divergent species to address evolutionary questions at a greater phylogenetic depth. Two in silico studies evaluated the suitability of RAD-tag sequencing to address phylogenetic questions (Rubin et al. 2012; Cariou et al. 2013) using simulated datasets obtained from divergent reference genomes. Rubin et al. (2012) used genomes from three taxonomic groups (Drosophila, mammals and yeasts) to generate RAD-tag sequences in silico and, for each group, assessed whether accurate species phylogenies could be reconstructed from these sequences. Similarly, Cariou et al. (2013) simulated RADtag sequences from the genomes of 12 species of Drosophila, separated by different levels of divergence ( 5 to $63 \mathrm{Mya}$ ). Both studies suggest that 1) a sufficient number (at least hundreds) of conserved orthologous loci can be obtained even when comparing divergent species within relatively young phylogenetic groups (divergence times of up to $60 \mathrm{Mya}$ ), and 2) RAD-tag loci can be phylogenetically informative and allow reconstruction of accurate species phylogenies.

Few empirical studies have evaluated whether these expectations are verified by including divergent species in their RAD-taq sequencing analysis (Eaton \& Ree 2013; Nadeau et al. 2013; Stölting et al. 2013), and particularly beyond intra-generic (Keller et al., 2012; Jones et al., 2013; Lexer et al., 2013; Wagner et al. 2013) or intra-familial (Bergey et al., 2013) comparisons. In the present study, we assessed 
the applicability of RAD-tag genotyping in the upper bound of these phylogenetic depths by conducting intra- and inter-familial comparisons using two cetacean species: the common dolphin (Delphinus delphis, Delphinidae) and the harbor porpoise (Phocoena phocoena, Phocoenidae). We analyzed generated RADtag sequences using the Stacks analysis pipeline (Catchen et al., 2011) and evaluated the effects of varying two Stacks parameters on the number of loci and genetic distances obtained.

\section{Materials and Methods}

\section{Tissue samples, DNA extraction and Sanger sequencing}

Tissue samples were collected from six dead animals (five short-beaked common dolphins, Delphinus delphis, and one harbor porpoise, Phocoena phocoena) that were either incidentally caught in pelagic fisheries in the Celtic Sea or Bay of Biscay, or stranded on the French Atlantic coast (Table 1). Tissue samples were frozen at $-20^{\circ} \mathrm{C}$ or stored in ethanol at room temperature. Total genomic DNA was extracted from approximately 15 to $25 \mathrm{mg}$ of skin or kidney tissue using NucleoSpin ${ }^{\circledR}$ Tissue (Macherey-Nagel EURL, Hoerdt, France) or using DNeasy ${ }^{\circledR}$ Blood \& Tissue (Qiagen, Courtaboeuf, France) kits following the manufacturer's protocols. DNA concentration was quantified using a NanoDrop ${ }^{T M} 2000$ (Thermo Scientific, Illkirch, France). DNA quality was assessed on a $1 \%$ agarose gel stained with ethidium bromide and was similar across the six samples: good (high molecular weight as well as shear) to excellent (high molecular weight only). Species identification made in the field using morphological characters was confirmed by sequencing two portions of the mitochondrial genome: 1) the 5' end of the control region (including a portion of the flanking proline tRNA) was amplified using primers L15824 (5'-CCTCACTCCTCCCTAAGACT3'; Rosel et al. 1999) and H16498 (5'-CCTGAAGTAAGAACCAGATG'-3; Rosel et al. 1994); 2) a portion of cytochrome $b$ was amplified using primers L14724 (5'-TGACTTGAARAACCAYCGTTG-3'; Palumbi et al. 1991) and H15149 (5'-CAGAATGATATTTGTCCTCA-3'; Kocher et al. 1989). The polymerase chain reaction (PCR) included $10 \mathrm{mM}$ Tris- $\mathrm{HCl}(\mathrm{pH} 8.3), 50 \mathrm{mM} \mathrm{KCl}, 0.1 \%$ Triton X-100, $1.5 \mathrm{mM}$ $\mathrm{MgCl}_{2}, 0.3 \mu \mathrm{M}$ of each primer, $0.15 \mathrm{mM}$ dNTPs (Euromedex, Mundolsheim, France), $2 \mathrm{U}$ Taq poly- 
merase (VWR, Fontenay sous Bois, France), and $50 \mathrm{ng}$ DNA in a $50 \mu \mathrm{L}$ total volume. PCR profiles were as described in Vollmer et al. (2011) for the L15824/H16498 primer pair and Viricel \& Rosel (2012) for the L14724/H15149 primer pair. PCR products were sent to Genoscreen (Lilles, France) for purification and Sanger sequencing. Mitochondrial sequences were edited using Sequencher ${ }^{\circledR}$ v. 4.7 (Gene Codes Corp., Ann Arbor, MI, USA) and were aligned using MAFFT v. 7 with default parameters (FFT-NS-i method) (Katoh et al., 2002).

\section{Genotyping by sequencing}

RAD-tag libraries were prepared by Eurofins Genomics (Ebersberg, Germany) using 1-2 $\mu \mathrm{g}$ of total genomic DNA per individual and using the Not1 restriction enzyme. Unique barcodes used to differentiate multiplexed individuals were six to nine nucleotides long and differed by at least two nucleotides. Libraries were sequenced by Eurofins Genomics on two lanes of the Illumina ${ }^{\circledR}$ HiSeq ${ }^{\top M} 2000$ platform (Illumina, Inc., San Diego, CA, USA) with the $1 \times 100$ base-pairs (bp) single-end read module, as part of a larger D. delphis population genomics RAD-tag sequencing project (total of 92 individuals). Raw Illumina reads were processed using the CASAVA v. 1.8.2 software (Illumina, Inc., San Diego, CA, USA). Illumina read data were de-multiplexed, quality-filtered and assembled using the Stacks tool kit v. 0.99994. A recent study by Davey et al. (2013) compared Stacks and RADtools (Baxter et al., 2011), another program to analyze RAD-tag sequences without a reference genome, and recommended use of Stacks as it provides more features. The Stacks pipeline includes four major steps (Catchen et al., 2013): reads are first sorted by unique barcode to group together all sequences from each individual (de-multiplexing step) while also excluding sequences that do not pass a set quality score; second, loci are build within each individual by creating stacks of identical reads and assembling unique loci by merging stacks that differ only by a set number of nucleotides (M) to allow polymorphism within individuals; third, loci identified for each individual are compared and catalogued across all individuals and a set number of nucleotide differences (n) is allowed to merge loci from different individuals in the catalog; fourth, individual genotypes are determined for each locus. The following filters were applied during the first step 
of the pipeline (process_radtags): one nucleotide mismatch (i.e. one sequencing error) was tolerated within individual barcodes, sequences for which the mean raw Phred quality score dropped below 10 within a sliding window spanning $15 \%$ of the read length were discarded. Sequences were truncated to a length of $91 \mathrm{bp}$. Since there is no reference genome available for D. delphis or P. phocoena, we used the denovo_map.pl program in Stacks to merge stacks (i.e., sets of identical reads) into loci within individuals and to build a catalog of loci across individuals. The minimum number of reads to form a stack (m) was set to 3. SNPs were detected while varying two Stacks parameters: the number of mismatches allowed between stacks to be grouped in a unique locus within an individual (M, set between 1 and 7), and the maximum distance among loci from distinct individuals to be merged in the population catalog (n, set between 1 and 8). Analyses were conducted with 14 different combinations of these parameters setting $\mathrm{M}$ and $\mathrm{n}$ at the same value or with one additional mismatch for $\mathrm{n}$ compared to $\mathrm{M}$ (see Figures 1 and 2). Highly repetitive sequences were removed or broken down using the 't' option in denovo_map.pl (Catchen et al., 2011, 2013). Additionally, we verified that the final catalog did not contain dimers formed by adapters. The quality of filtered sequences obtained after denovo_map.pl was evaluated using FastQC v. 0.10.1 (Babraham Bioinformatics, www.bioinformatics.babraham.ac.uk/projects/). We applied the populations program from Stacks to obtain the final sets of orthologous loci: loci were retained if the locus total depth of coverage was equal or higher than 10 reads per individual, and if they were present in all individuals (i.e. no missing data allowed).

\section{Data analyses}

Polymorphism and divergence statistics were calculated using the populations program in Stacks, and using the ape (Paradis et al., 2004) and adegenet (Jombart, 2008) packages in R (R Development Core Team, 2013), respectively. Inter-individual divergence was assessed using polymorphic sites that are either variable within individuals (in heterozygotes), or fixed within individuals (homozygotes) but variable between individuals. Due to likely heterogeneity in substitution models across loci, genetic distances were calculated as raw p-distances (i.e., proportion of fixed differences between two sequences). 
To compare intra-specific and inter-familial data, all analyses were run on: 1) the five Delphinus delphis individuals, and 2) all six individuals (five D. delphis and one Phocoena phocoena).

Finally, for the inter-familial comparison, we explored the functions of invariable (conserved) compared to polymorphic loci, at a chosen $\mathrm{M}$ and $\mathrm{n}$ combination (M3n3; see Results section). The goal of this analysis was two-tiered: 1) to investigate whether some functions would be overrepresented in polymorphic compared to invariable loci, 2) to assess whether putative gene functions can be retrieved from RADtag sequences, which could be useful in applications such as studying loci potentially under selection. Identification of RAD tag sequences (1,587 and 3,574 tags for conserved and variable loci, respectively) was determined in Blast2GO v. 2.6 .6 (public database of August 2012; Conesa et al., 2005; Conesa \& Götz, 2008; Götz et al., 2011, 2008) using the blastn program (e-value cut off of 10-3, HSP cut-off of 33 Altschul et al., 1990, 1997), as the blastx program retrieved very little results due to the short length of the corresponding amino-acid sequences ( $<31$ amino-acids). While blastn can be used to identify tags, it does not allow subsequent mapping and annotation (Blast2GO manual). We therefore used the results of blastn to retrieve the sequence of the best match between tags and Genbank sequences from the Tursiops truncatus genome (Genbank Bioprojects accession numbers PRJNA189944 and PRJNA20367), a species closely related to $D$. delphis (McGowen et al., 2009), similar to the approach employed by Reitzel et al. (2013) for the anemone Nematostella vectensis. As the percent identity between tags and T. truncatus sequences was very high (84.6 to $100 \%)$, we proceeded to the mapping and annotation steps to obtain Gene Ontology (GO) terms from these longer T. truncatus sequences (222 to 16,700 bp, median size 1,549 bp; blastx e-value cutoff $=10^{-3} \mathrm{HSP}=33,20$ hits retained; annotation settings: e-value filter $=10^{-6}$, annotation cutoff $=55, \mathrm{GO}$ weight $=5$, no HSP-hit coverage cutoff). GO terms correspond to groups of genes involved in similar functions such as genes with products involved in cellular components. Genes can be grouped into GO terms at different levels depending on the desired level of precision in the function. Enrichment of GO terms between T. truncatus sequence sets corresponding to "conserved" and "variable" loci were tested using the Fisher's Exact test as implemented in Blast2GO (GOSSIP module, 
155

\section{Results}

Species identification of each individual was confirmed using Sanger-sequenced mitochondrial DNA. Mitochondrial sequence alignments encompassed a 425 bp portion of the control region and a 402 bp portion of cytochrome $b$ (see Table 1 for Genbank accession numbers). For the five common dolphins, control region sequences were identical to haplotypes published on Genbank (i.e. 100\% coverage and 100\% identity), which were sequenced from other short-beaked common dolphins from the eastern North Atlantic (NA). Cytochrome $b$ sequences for these individuals also supported species identification made in the field as most similar sequences in Genbank belonged to short-beaked common dolphins. For the harbor porpoise, we obtained a perfect haplotype match for the mitochondrial control region sequence, corresponding to another harbor porpoise from the eastern NA. For cytochrome $b$, there was a one bp difference between our sequence and a published haplotype (accession number: AJ554063) from a harbor porpoise complete mitogenome. The next best match in Genbank was also a harbor porpoise (accession number: U13143).

The two Illumina sequencing lanes produced over 2.8 million raw reads per individual (Table 2). On average, $40 \%$ of raw reads were removed by the quality filters applied (Table 2). The main reason for removing reads was ambiguous barcodes, which could suggest either barcode synthesis errors, or a high sequencing error rate. By setting the minimum number of reads to build a stack to 3 , the impact of potential sequencing errors on the genotypes and loci we obtained should be very limited. The sequence quality of filtered reads was excellent with a minimum Phred score of 35 (Table 2).

The effect of varying the denovo_map.pl parameters $M$ and $n$ can be contrasted between the intraspecific and inter-familial datasets. For the inter-familial comparison, increasing M (intra-individual parameter), and particularly n (inter-individual parameter) resulted in an increase in the total number of loci until a plateau was reached at parameter combination M3n3 (Figure 1a; total number of loci: 5182). This outcome can be explained as follows: in the first step of the analysis (i.e., denovo_map.pl), 
increasing $\mathrm{M}$ and $\mathrm{n}$ will decrease the total number of loci present in the catalog as more distinct sequences will be merged into the same locus (Catchen et al. 2013). This also results in a greater depth of coverage per locus. Thus, in the second step of the analysis (populations), there will actually be an increase in the total number of loci that are kept in the final catalog after the filters are applied (a minimum of 10 reads per locus per individual). Additionally, as $\mathrm{n}$ is increased, more loci will be in common among all individuals, particularly when including divergent individuals such as here. When $\mathrm{n}$ is low, fixed differences will be considered as distinct loci that will not be present in all individuals. Therefore, the number of loci kept in the final catalog will also increase when $\mathrm{n}$ is increased due to the filter of the minimum number of individuals where a locus has to be present (in this study, a locus had to be present in all individuals). A similar trend can be observed for the number of polymorphic loci, which increased as $\mathrm{M}$ and $\mathrm{n}$ increased (Figure $1 \mathrm{~b}$ ). Eventually, increasing $\mathrm{M}$ and $\mathrm{n}$ could result in overmerging loci (loci that are not orthologous). By using the set of parameters corresponding to where a plateau for the total number of loci starts, we were most likely to avoid overmerging issues. However, it is possible that overmerging is not detectable by simply observing a plateau in the total number of loci, as the overall decrease in the number of loci by overmerging could be balanced by the discovery of orthologous, yet highly divergent loci. For the intra-specific dataset, the effect of varying the two parameters on the number of loci (total and polymorphic) was less striking (Figures 1a,b). A plateau was quickly reached at the M2n2 parameter combination (total number of loci: 7838; 2032 polymophic loci) after an initial small rise in the number of polymorphic loci (Figure 1b). In terms of divergence, the largest change for both datasets was observed when increasing $\mathrm{n}$ from 1 to 2 (Figure 2a,b). This is likely due to an increase in the number of variable sites that are fixed within but variable among individuals. For subsequent data description (e.g. sequence variability) and analyses (Blast2GO), we chose the parameter combinations where a plateau was reached in terms of number of loci, which corresponded to M3n3 for the inter-familial dataset and M2n2 for the intra-specific dataset.

Using these parameter combinations, we observed a 34\% drop in the total number of loci when 
analyzing all individuals (in the inter-familial comparison) compared to the intra-specific dataset. This drop was calculated as the percent difference in the total number of loci found with and without the $P$. phocoena sample in the final dataset. This result was not simply an effect of removing any individual from the dataset, as excluding a $D$. delphis individual only resulted in a small percent difference (2.2 to $6.5 \%$ ) in the total number of loci. Thus, we can conclude that most of the $34 \%$ percent drop in number of loci was indeed due to including a more divergent individual. The proportion of variable loci was $69 \%$ and $26 \%$ for the inter-familial and intra-specific datasets, respectively. In terms of sequence polymorphism, we observed one SNP every 292 bp in the intra-specific dataset (total sequence length screened: $713,255 \mathrm{bp}$ ) compared to one SNP every $71 \mathrm{bp}$ in the inter-familial dataset (total sequence length screened: $471,538 \mathrm{bp})$.

In the inter-familial comparison, sequence identification using Blast2GO was significantly higher for conserved tags $(994 / 1587,63 \%)$ than for polymorphic tags $(1512 / 3574,42 \%)$. blastn searches were reliable, with e-values ranging from $10^{-5}$ to $10^{-38}$ for the best hit. Similarity between query and subject sequences of the best hit was high (73-100\%, median $100 \%$ for conserved tags; $71-100 \%$, median $98 \%$ for polymorphic tags). Top species hits included sequences from killer whale (Orcinus orca: 774 hits), common bottlenose dolphin (Tursiops truncatus: 716 hits), human (Homo sapiens: 234 hits), and other mammals.

A majority of the best hit sequences from T. truncatus could be mapped and annotated (conserved: 91\%; variable: 92\%). There was no significant difference in the number of T. truncatus sequences per GO term between variable versus conserved loci across the two cetacean families (Fisher's exact test with FDR $=0.05 ;$ Figure S1, supporting information).

\section{Discussion}

Modern cetaceans constitute a recent group, which originated approximately 34 to 35 mya (Fordyce, 1980; Arnason et al., 2004) and comprise 14 extant families (Perrin et al., 2009). Thus, based on the 
two in silico studies from Rubin et al. (2012) and Cariou et al. (2013), a large number of conserved orthologous loci should be obtained when comparing species from distinct cetacean families. Indeed, our study confirms this expectation, as only $34 \%$ of the loci present, at this sequencing effort, in all common dolphins were lost when including an individual from a distinct cetacean family. The divergence time separating these two families (Delphinidae and Phocoenidae) has been estimated between 14 and 19 Mya based on fossil-calibrated molecular clocks (Arnason et al., 2004; Xiong et al., 2009; McGowen et al., 2009). Comparatively, the proportion of loci that were lost (34\%) in our inter-familial comparison was lower than the percentage of loci lost $(60 \%)$ between two divergent Drosophila species pairs, which have been separated for a similar period of time (ca 13 My) (Cariou et al., 2013). At similar divergence times, the loss of orthologous loci will depend on the rate of molecular evolution, which varies among taxonomic groups (Britten, 1986; Martin \& Palumbi, 1993). Indeed, Drosophila has a high nucleotide substitution rate (e.g. Britten, 1986; Chan et al., 2012) compared to cetaceans, which generally display slow rates of molecular evolution (Kingston \& Rosel, 2004; Bininda-Emonds, 2007; McGowen et al., 2012).

We explored the effects of varying two parameters in the denovo_map.pl program of Stacks on the number of loci and level of divergence obtained. A plateau was reached, after which the number of loci did not change dramatically. Our results are comparable to Keller et al. (2012) who observed a decrease in the total number of loci obtained when increasing $\mathrm{M}$ and $\mathrm{n}$, prior to applying filters, and an increase in the number of polymorphic loci after filters were applied. The level of sequence variability we observed in intra-specific comparisons (within D. delphis: one SNP every $292 \mathrm{bp}$ ) was comparable to the diversity previously observed in Delphinus spp. or in closely related species. Thus, Amaral et al. (2010) screened 6,537 bp in 17 Delphinus spp. individuals and reported a SNP every 272 bp. For the common bottlenose dolphin (Tursiops truncatus), Vollmer \& Rosel (2012) observed one SNP every 463 bp (total sequence length screened: 70,828 bp in 10 individuals). Note that the five common dolphins analyzed here were from the eastern NA, and possibly from the same population. Greater sequence variability would be expected if individuals from distinct regions or populations were analyzed as in Vollmer \& Rosel (2012) 
and Amaral et al. (2010). A source of sequence variability that we did not consider here is the occurrence of indels. Stacks does not allow for indels and these sequences would appear as distinct loci in our analysis and would not pass the filter of being present in all individuals. Therefore, we may have lost some loci if indels were present. Among alternative pipelines that have been developed to analyze RADtag data in absence of a reference genome, PyRAD (Eaton \& Ree, 2013) does accommodate indels. PyRAD is based on sequence similarity and alignment, rather than a set number of nucleotide differences.

The number of polymorphic loci and sequence variability (one SNP every $71 \mathrm{bp}$ ) observed in our interfamilial comparison outline the potential benefits of RAD-tag sequencing to solve phylogenetic questions within a group that diversified in multiple rapid and recent radiation events (Steeman et al., 2009). Recently, analysis of amplified fragment length polymorphisms (AFLPs) has provided new insights into the phylogeny of cetaceans (Kingston \& Rosel, 2004; Kingston et al., 2009). However, these markers are dominant and anonymous. One advantage of RAD-tag sequencing compared to the approach above is that it provides co-dominant sequence data, which can be potentially identified and annotated using published databases (e.g. Scaglione et al., 2012). Our Blast2GO results suggest that insights into putative function can be gained by comparing short ( $<100$ bp) RAD-tag sequences to published sequences. However, GO terms associated with RAD sequences could not be obtained directly using blastx due to the short length of the corresponding amino-acid sequences and Blast2GO does not produce GO terms when blastn is applied. Thus, obtaining GO terms was achieved indirectly by relying on blastn hits from the reference genome of a closely related species ( T. truncatus) and applying blastx on these longer sequences. While we limited our phylogenetic analyses to comparing GO terms between conserved and polymophic loci, and calculating genetic distances, Stacks produces outputs that allow to conduct other analyses widely-used in phylogeny or phylogeography such as building phylogenetic trees and running cluster analyses.

One limitation of RAD-tag sequencing for phylogenetic inferences is that the number of loci is expected to decrease as more taxa are added to the dataset (as seen in Lexer et al., 2013), which will limit to some extent the size of the phylogenetic dataset. Thus, there will be a trade-off between the number of taxa and 
the total number of orthologous loci analyzed. One way to alleviate this issue could be to use a RADtag double-digest approach (Peterson et al., 2012), which would increase locus representation across individuals. Very recently, new high-throughput genomic sequencing approaches have been developed to specifically target phylogenomics and phylogeographic questions (Carstens et al., 2012; Lemmon \& Lemmon, 2013). These new laboratory methods should be complementary to applications of RADseq data. A first approach, developed by Lemmon et al. (2012), is based on a sequence capture technique, which relies on probes designed using sequenced reference genomes. This approach, termed anchored enrichment, can provide several hundreds of loci, in the form of sequence data, for potentially hundreds of individuals from model and non-model organisms and should be applicable at different phylogenetic depths. An advantage of this approach, is that it should be applicable to degraded samples. To date however, its applicability to recently and rapidly evolved groups has not been empirically assessed yet. A second approach was designed to investigate relationships at greater phylogenetic depths by targeting ultraconserved elements (Faircloth et al., 2012; McCormack et al., 2012). While RAD-tag sequencing may be more appropriate for questions related to species delimitation and phylogeography in rapidly and recently diverged groups (e.g., cetaceans), other approaches such as anchored enrichment should be used when the phylogenetic depth in question reaches the limits of utility of RAD-tag data (for a full comparison of these methods, see review by Lemmon \& Lemmon, 2013). In the near future, we should gain a better sense of the limits of each approach as studies implementing these new methods accumulate.

In conclusion, our empirical study supports expectations that the applicability of RAD-tag genotyping is not limited to closely related species. Using two mammalian species from distinct, but recently evolved, families, we showed that this approach holds great promise for evolutionary studies conducted at this phylogenetic level. 


\section{Acknowledgments}

We thank the following people and institutions for collecting and providing tissue samples: Oliver van Canneyt, Fabien Demaret and Ghislain Dorémus (UMS Pelagis) and the French stranding network (RNE). DNA samples were prepared at the Molecular Core Facility at the University of La Rochelle. We thank Vanessa Becquet (University of La Rochelle) for laboratory assistance. We would like to acknowledge Bruce Deagle, and three anonymous reviewers for constructive comments on the manuscript. The University of La Rochelle supercomputer "YMIR" was used to run Stacks and was partly funded by the European Regional Development Fund. We thank Mikael Guichard for his help with "YMIR." This work was funded by LIENSs; salaries for AV and EP were covered by a grant to the Poitou-Charentes region (Contrat de Projet État-Région 2007-2013), by a grant from the Fond Européen de Développement Régional (EP), and by Actions internationales et rayonnement of the University of La Rochelle (AV).

\section{References}

Altschul S, Gish W, Miller W, Myers E, Lipman D (1990) Basic local alignment search tool. Journal of Molecular Biology, 215, 403-410.

Altschul SF, Madden TL, Schäffer AA, et al. (1997) Gapped BLAST and PSI-BLAST: a new generation of protein database search programs. Nucleic Acids Research, 25, 3389-3402.

Amaral AR, Silva MC, Möller LM, Beheregaray LB, Coelho MM (2010) Anonymous nuclear markers for cetacean species. Conservation Genetics, 11, 1143-1146.

Arnason U, Gullberg A, Janke A (2004) Mitogenomic analysis provide new insight into cetacean origin and evolution. Gene, 333, 27-34.

Baxter SW, Davey JW, Johnston JS, et al. (2011) Linkage mapping and comparative genomics using next-generation rad sequencing of a non-model organism. PLoS One, 6, e19315. 
Bergey CM, Pozzi L, Disotell TR, Burrell AS (2013) A new method for genome-wide marker development and genotyping holds great promise for molecular primatology. International Journal of Primatology, 34, 303-314.

Bininda-Emonds ORP (2007) Fast genes and slow clades: comparative rates of molecular evolution in mammals. Evolutionary Bioinformatics, 3, 59-85.

Blüthgen N, Brand K, Cajavec B, Swat M, Herzel H, Beule D (2005) Biological profiling of gene groups utilizing gene ontology. Genome Inform, 16, 106-115.

Britten RJ (1986) Rates of DNA sequence evolution differ between taxonomic groups. Science, 231, $1393-1398$.

Cariou M, Duret L, Charlat S (2013) Is RAD-seq suitable for phylogenetic inference? An in silico assessment and optimization. Ecology and Evolution, 3, 846-852.

Carstens B, Lemmon AR, Lemmon EM (2012) The promises and pitfalls of next-generation sequencing data in phylogeography. Systematic Biology, 61, 713-715.

Catchen J, Hohenlohe PA, Bassham S, Amores A, Cresko WA (2013) Stacks: an analysis tool set for population genomics. Molecular Ecology, 22, 3124-3140.

Catchen JM, Amores A, Hohenlohe P, Cresko W, Postlethwait JH (2011) Stacks: building and genotyping loci de novo from short-read sequences. G3 (Bethesda), 1, 171-82.

Chan AH, Jenkins PA, Song YS (2012) Genome-wide fine-scale recombination rate variation in Drosophila melanogaster. PLoS Genetics, 8, e1003090.

Conesa A, Götz S (2008) Blast2go: A comprehensive suite for functional analysis in plant genomics. International Journal of Plant Genomics, Article ID 619832, 12 pages. 
Conesa A, Götz S, García-Gómez JM, Terol J, Talón M, Robles M (2005) Blast2GO: a universal tool for annotation, visualization and analysis in functional genomics research. Bioinformatics, 21, 3674-3676.

Davey JW, Cezard T, Fuentes-Utrilla P, Eland C, Gharbi K, Blaxter ML (2013) Special features of RAD sequencing data: implications for genotyping. Molecular Ecology, 22, 3151-3164.

Eaton DAR, Ree RH (2013) Inferring phylogeny and introgression using RADseq data: an example from flowering plants (Pedicularis: Orobanchaceae). Systematic Biology, 0, 1-18.

Emerson KJ, Merz CR, Catchen JM, et al. (2010) Resolving postglacial phylogeography using highthroughput sequencing. Proc Natl Acad Sci U S A, 107, 16196-200.

Faircloth BC, McCormack JE, Crawford NG, Harvey MG, Brumfield RT, Glenn TC (2012) Ultraconserved elements anchor thousands of genetic markers spanning multiple evolutionary timescales. Systematic Biology, 61, 717-726.

Fordyce RE (1980) Whale evolution and Oligocene Southern Ocean environments. Palaeogeography, Palaeoclimatology, Palaeoecology, 31, 319-336.

Gagnaire PA, Normandeau E, Pavey SA, Bernatchez L (2013) Mapping phenotypic, expression and transmission ratio distortion QTL using RAD markers in the Lake Whitefish (Coregonus clupeaformis). Molecular Ecology, 22, 3036-3048.

Götz S, Arnold R, Sebastián-León P, et al. (2011) B2G-FAR, a species-centered GO annotation repository. Bioinformatics, 27, 919-924.

Götz S, Garciá-Gómez JM, Terol J, et al. (2008) High-throughput functional annotation and data mining with the Blast2GO suite. Nucleic Acids Research, 36, 3420-3435.

Hohenlohe PA, Amish SJ, Catchen JM, Allendorf FW, Luikart G (2011) Next-generation RAD sequencing identifies thousands of SNPs for assessing hybridization between rainbow and westslope cutthroat trout. Molecular Ecology Resources, 11 Suppl 1, 117-22. 
Jombart T (2008) adegenet: a R package for the multivariate analysis of genetic markers. Bioinformatics, 24, 1403-5.

Jones JC, Fan S, Franchini P, Schartl M, Meyer A (2013) The evolutionary history of Xiphophorus fish and their sexually selected sword: a genome-wide approach using restriction site-associated DNA sequencing. Molecular Ecology, 22, 2986-3001.

Katoh K, Misawa K, Kuma K, Miyata T (2002) MAFFT: a novel method for rapid multiple sequence alignment based on fast Fourier transform. Nucleic Acids Research, 30, 3059-3066.

Keller I, Wagner CE, Greuter L, et al. (2012) Population genomic signatures of divergent adaptation, gene flow and hybrid speciation in the rapid radiation of Lake Victoria cichlid fishes. Molecular Ecology.

Kingston SE, Adams LD, Rosel PE (2009) Testing mitochondrial sequences and anonymous nuclear markers for phylogeny reconstruction in a rapidly radiating group: molecular systematics of the Delphininae (Cetacea: Odontoceti: Delphinidae). BMC Evolutionary Biology, 9, 245.

Kingston SE, Rosel PE (2004) Genetic differentiation among recently diverged delphinid taxa determined using AFLP markers. Journal of Heredity, 95, 1-10.

Kocher TD, Thomas WK, Meyer A, et al. (1989) Dynamics of mitochondrial DNA evolution in animals: Amplification and sequencing with conserved primers. Proceedings of the National Academy of Sciences of the United States of America, 86, 6196-6200.

Lemmon AR, Emme SA, Lemmon EM (2012) Anchored hybrid enrichment for massively high-throughput phylogenomics. Systematic Biology, 61, 727-744.

Lemmon EM, Lemmon AR (2013) High-throughput genomic data in systematics and phylogenetics. Annual Review of Ecology, Evolution, and Systematics, 44, 19.1-19.23.

Lexer C, Mangili S, Bossolini E, et al. (2013) 'Next generation' biogeography: towards understanding the drivers of species diversification and persistence. Journal of Biogeography, 40, 1013-1022. 
Martin AP, Palumbi SR (1993) Body size, metabolic rate, generation time, and the molecular clock. Proceedings of the National Academy of Sciences of the United States of America, 90, 4087-4091.

McCormack JE, Faircloth BC, Crawford NG, Gowaty PA, Brumfield RT, Glenn TC (2012) Ultraconserved elements are novel phylogenomic markers that resolve placental mammal phylogeny when combined with species-tree analysis. Genome Research, 22, 746-754.

McCormack JE, Hird SM, Zellmer AJ, Carstens BC, Brumfield RT (2013) Applications of next-generation sequencing to phylogeography and phylogenetics. Mol Phylogenet Evol, 66, 526-538.

McGowen MR, Grossman LI, Wildman DE (2012) Dolphin genome provides evidence for adaptive evolution of nervous system genes and a molecular rate slowdown. Proceedings of the Royal Society B-Biological Sciences, 279, 3643-3651.

McGowen MR, Spaulding M, Gatesy J (2009) Divergence date estimation and a comprehensive molecular tree of extant cetaceans. Molecular Phylogenetics and Evolution, 53, 891-906.

Nadeau NJ, Martin SH, Kozak KM, et al. (2013) Genome-wide patterns of divergence and gene flow across a butterfly radiation. Molecular Ecology, 22, 814-826.

Palumbi S, Martin A, Romano S, McMillan W, Stice L, Grabowski G (1991) The simple fool's guide to PCR. version 2.0. Tech. Rep., University of Hawaii, Honolulu.

Paradis E, Claude J, Strimmer K (2004) APE: analyses of phylogenetics and evolution in R language. Bioinformatics, 20, 289-290.

Perrin W, Würzig B, Thewissen J, eds. (2009) Encyclopedia of Marine Mammals, Second Edition. Academic Press, San Diego.

Peterson BK, Weber JN, Kay EH, Fisher HS, Hoekstra HE (2012) Double digest RADseq: an inexpensive method for de novo SNP discovery and genotyping in model and non-model species. PLoS One, 7, e37135. 
R Development Core Team (2013) R: A Language and Environment for Statistical Computing. R Foundation for Statistical Computing, Vienna, Austria. ISBN 3-900051-07-0.

Reitzel AM, Herrera S, Layden MJ, Martindale MQ, Shank TM (2013) Going where traditional markers have not gone before: utility of and promise for rad sequencing in marine invertebrate phylogeography and population genomics. Molecular Ecology, 22, 2953-2970.

Rosel PE, Dizon AE, Heyning JE (1994) Genetic-analysis of sympatric morphotypes of common dolphins (genus Delphinus). Marine Biology, 119, 159-167.

Rosel PE, Tiedemann R, Walton M (1999) Genetic evidence for limited trans-Atlantic movements of the harbor porpoise Phocoena phocoena. Marine Biology, 133, 583-591.

Rubin BER, Ree RH, Moreau CS (2012) Inferring phylogenies from RAD sequence data. PLoS One, 7, e33394.

Scaglione D, Acquadro A, Portis E, Tirone M, Knapp SJ, Lanteri S (2012) RAD tag sequencing as a source of SNP markers in Cynara cardunculus L. BMC Genomics, 13, 3.

Stapley J, Reger J, Feulner PDG, et al. (2010) Adaptation genomics: the next generation. Trends in Ecology \& Evolution, 25, 705-712.

Steeman ME, Hebsgaard MB, Fordyce RE, et al. (2009) Radiation of extant cetaceans driven by restructuring of the oceans. Systematic Biology, 58, 573-585.

Stölting KN, Nipper R, Lindtke D, et al. (2013) Genomic scan for single nucleotide polymorphisms reveals patterns of divergence and gene flow between ecologically divergent species. Molecular Ecology, 22, $842-855$.

Viricel A, Rosel PE (2012) Evaluating the utility of cox1 for cetacean species identification. Marine Mammal Science, 28, 37-62. 

truncatus): isolation and characterization of 153 single nulceotide polymorphisms and 53 genotyping assays. Molecular Ecology Resources, 12, 1124-1132. in multiple cetacean species. Current Genetics, 57, 115-131.

Wagner CE, Keller I, Wittwer S, et al. (2013) Genome-wide rad sequence data provide unprecedented res-

olution of species boundaries and relationships in the lake victoria cichlid adaptive radiation. Molecular Ecology, 22, 787-798.

Xiong Y, Brandley MC, Xu S, Zhou K, Yang G (2009) Seven new dolphin mitochondrial genomes and a time-calibrated phylogeny of whales. BMC Evolutionary Biology, 9, 20.

\section{Data Accessibility}

Mitochondrial DNA sequences have been submitted to Genbank (see Table 1 for accession numbers).

Demultiplexed and filtered (i.e. after process-radtags) sequences (.fq files), R and Stacks codes, and Blast2G0 output files were deposited in Dryad (doi:10.5061/dryad.mk364).

\section{Author Contributions}

A.V. and B.S. designed the research; W.D. provided tissue samples and voucher information; A.V. conducted laboratory work; A.V. and E.P analyzed the data; A.V. and E.P wrote the manuscript. 

Figure Legends

451

Fig. 1 Influence of Stacks parameters on (a) the total number of loci, and (b) the number of polymorphic loci obtained. Fourteen parameter combinations were evaluated for the whole dataset (inter-familial comparison), and for the common dolphin only (intra-specific comparison).

Fig. 2 Influence of Stacks parameters on inter-individual sequence divergence (raw p-distances calculated using variable sites) for (a) inter-familial comparisons, and (b) intra-specific comparisons. The range of inter-individual distances is represented as boxplots. 
Table 1 Voucher information for one harbor porpoise (Phocoena phocoena) and five short-beaked common dolphins (Delphinus delphis) used in this study. Voucher identification (ID) numbers correspond to specimen numbers from UMS Pelagis. For samples obtained from stranded animals, the geographic coordinates and date of the stranding event are given. For one common dolphin that was incidentally caught (bycatch) in the tuna fishery, geographic coordinates and date correspond to where and when the dead animal was retrieved from the gear onboard. Genbank accession numbers are given for each mitochondrial DNA portion that was sequenced: control region (CR) and cytochrome $b(c y t b)$.

\begin{tabular}{|c|c|c|c|c|c|c|c|c|}
\hline Species & Voucher ID & Sample type & Sex & Latitude & Longitude & Date & CR accession no & cytb accession no \\
\hline Phocoena phocoena & 10712131 & bycatch & $\mathrm{M}$ & 44.648 & -1.316 & 13-Apr-07 & KF727592 & KF727598 \\
\hline Delphinus delphis & 10307073 & stranding & $\mathrm{F}$ & 46.713 & -1.979 & 29-Jul-03 & KF727593 & KF727599 \\
\hline Delphinus delphis & 10401011 & stranding & $\mathrm{F}$ & 44.404 & -1.264 & 17-Jan-04 & KF727594 & KF727600 \\
\hline Delphinus delphis & 10512077 & bycatch & $\mathrm{F}$ & 48.100 & -9.867 & 3-Sep-05 & KF727595 & KF727601 \\
\hline Delphinus delphis & 9902012 & stranding & M & 43.955 & -1.363 & 19-Feb-99 & KF727596 & KF727602 \\
\hline Delphinus delphis & 10201010 & stranding & $\mathrm{F}$ & 46.189 & -1.429 & 22-Jan-02 & KF727597 & KF727603 \\
\hline
\end{tabular}


Table 2 Total number of raw and filtered (i.e. after process-radtags) reads for each sample used in this study. Overall sequence quality was assessed using the mean Phred score after filters from process-radtags

\begin{tabular}{rrrrr} 
Species & Voucher ID & Raw reads & Filtered reads & Mean Phred score \\
\hline Phocoena phocoena & 10712131 & $3,102,559$ & $2,455,577$ & 36 \\
Delphinus delphis & 10307073 & $3,125,400$ & $1,614,204$ & 36 \\
Delphinus delphis & 10401011 & $3,185,527$ & $1,846,015$ & 36 \\
Delphinus delphis & 10512077 & $2,814,062$ & $2,091,276$ & 36 \\
Delphinus delphis & 9902012 & $3,859,005$ & $1,466,233$ & 36 \\
Delphinus delphis & 10201010 & $3,712,290$ & $2,105,664$ & 35 \\
\hline
\end{tabular}


Fig. 1

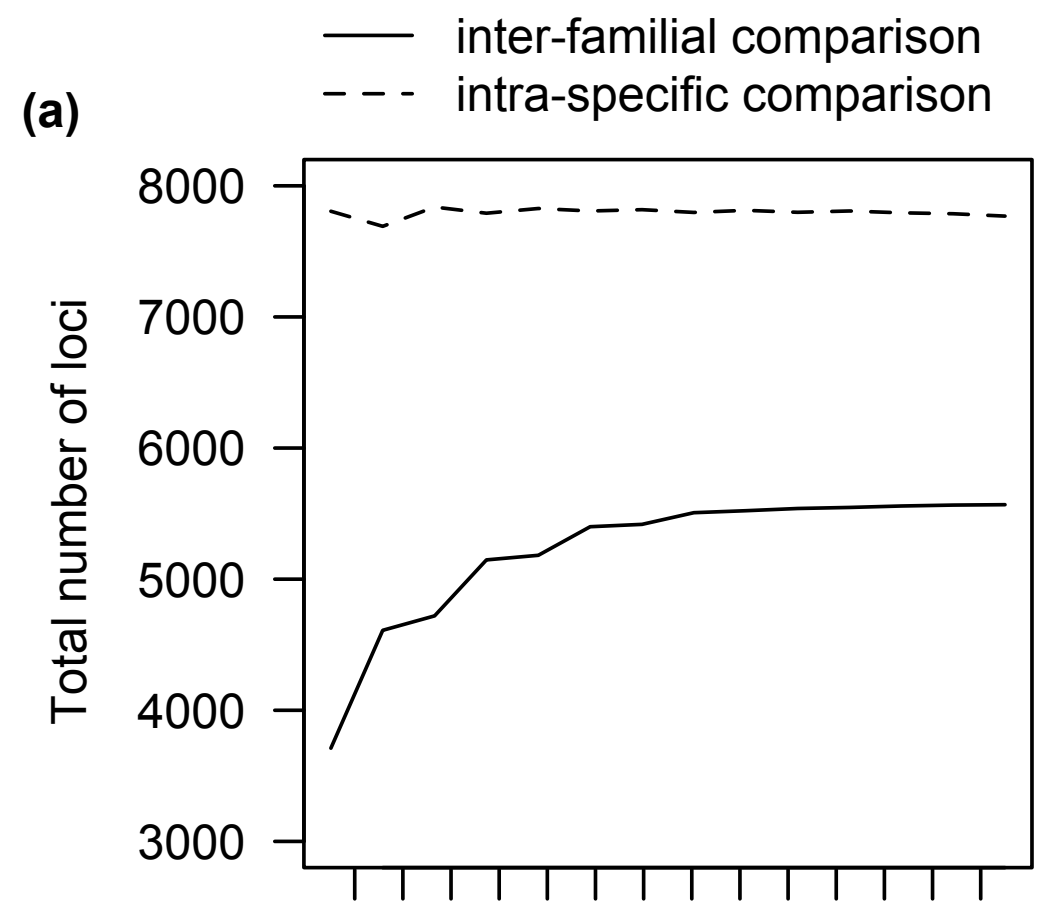

(b)

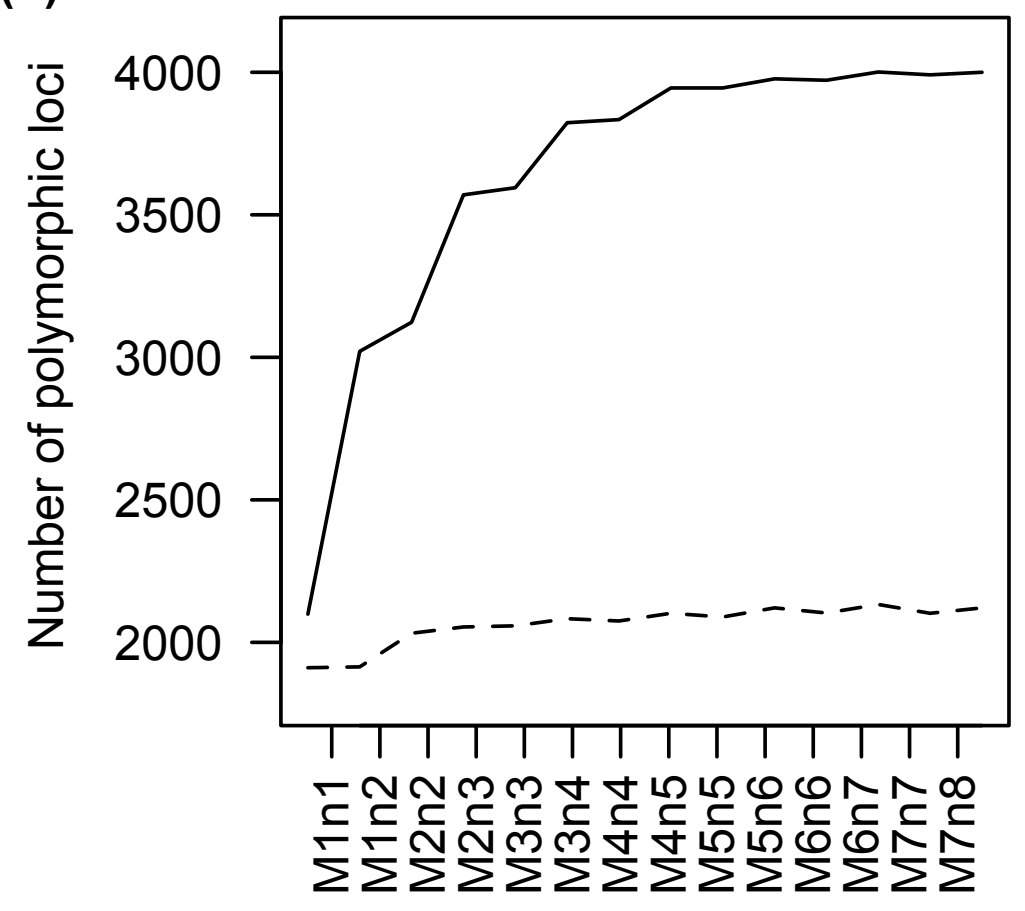




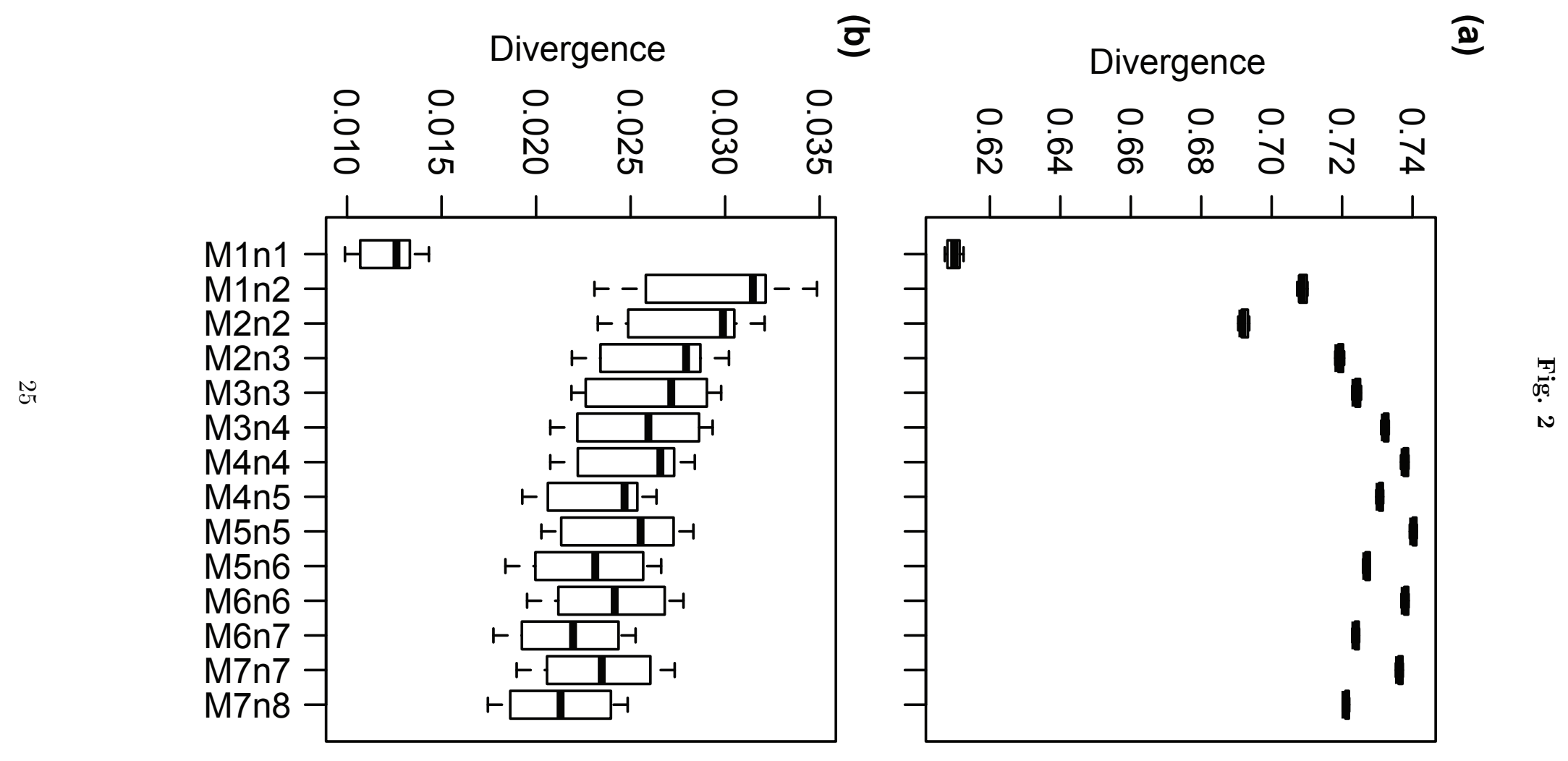

